\title{
CRECIMIENTO Y ESTABLECIMIENTO DE PLÁNTULAS DE Bouteloua gracilis (Kunth) Lag. ex Griffiths Y Eragrostis curvula var. conferta Stapf BAJO UN RÉGIMEN SIMULADO DE LLUVIA
}

\author{
GROWTH AND ESTABLISHMENT OF Bouteloua gracilis (Kunth) Lag. ex Griffiths \\ AND Eragrostis curvula var. conferta Stapf SEEDLINGS UNDER A SIMULATED RAINFALL REGIME
}

\author{
Blanca Moreno-Gómez ${ }^{1}$, Edmundo García-Moya ${ }^{2}$, Quintín Rascón-Cruz ${ }^{3}$ \\ y Gerardo A. Aguado-Santacruz ${ }^{1 \star}$
}

\begin{abstract}
${ }^{1}$ Laboratorio de Biotecnología y Fisiología Molecular de Plantas y Microorganismos(LABIFIPyM), Campo Experimental Bajío, Instituto Nacional de Investigaciones Forestales, Agrícolas y Pecuarias. Km 6.5 Carretera Celaya-San Miguel de Allende. 38110 , Celaya, Gto. México. ${ }^{2}$ Postgrado en Botánica, Colegio de Postgraduados. Km 36.5 Carretera México-Texcoco. 56230, Montecillo, Texcoco, Edo. México México. ${ }^{3}$ Facultad de Ciencias Químicas, Universidad Autónoma de Chihuahua. Circuito No.1 Nuevo Campus Universitario. 31125, Chihuahua, Chih. México.
\end{abstract}

*Autor para correspondencia (gaguado@prodigy.net.mx, gaguados@yahoo.com)

\section{RESUMEN}

Se evaluó el efecto de un régimen simulado de lluvia sobre el crecimiento y establecimiento de plántulas de los pastos navajita azul (Bouteloua gracilis) y boer (Eragrostis curvula var. conferta) bajo condiciones de invernadero. Un grupo de plántulas se mantuvo bajo condiciones de humedad adecuada mediante riegos a capacidad de campo, mientras que otro grupo de plántulas se mantuvo bajo un régimen simulado de lluvia que se derivó de los registros de una estación climática cercana al lugar de colecta de la semilla. Cada $3 \mathrm{~d}$ se midió el número de hojas, de vástagos secundarios y la altura de cada plántula, y al final $(37$ d) se determinó el porcentaje de establecimiento de plántulas, los pesos secos de vástago y raíz, y las relaciones raíz/vástago. Las plántulas de B. gracilis desarrollaron más vástagos secundarios y mayor altura bajo el régimen de capacidad de campo que en el régimen simulado de lluvia $(P \leq 0.05)$. Contrariamente, las plántulas de $E$. curvula crecidas bajo el régimen simulado de lluvia desarrollaron mayor cantidad de vástagos y de hojas que sus contrapartes crecidas bajo capacidad de campo y que $B$. gracilis bajo las dos condiciones de riego. Ambos pastos mostraron $90 \%$ de establecimiento bajo condiciones de capacidad campo, pero bajo el régimen simulado de lluvia esta variable disminuyó en $10 \%$ en el pasto boer. El esquema de evaluación aquí propuesto puede ser una herramienta confiable para analizar el establecimiento y desarrollo de plántulas de pastos bajo condiciones de invernadero y estrés hídrico.

Palabras clave: Bouteloua gracilis, Eragrostis curvula, estrés hídrico, capacidad de campo, invernadero.

\section{SUMMARY}

The effect of a simulated-rain regime was evaluated on the development and seedling establishment of blue grama Bouteloua gracilis, and boer lovegrass (Eragrostis curvula var. conferta) under greenhouse conditions. A group of seedlings was maintained under waterings to field capacity while another group of seedlings was maintained under a simulated-rain regime; the regime was simulated from real climate records registered in a weather station located near the site where the seeds were collected. Every $3 \mathrm{~d}$, the number of leaves, tillers, and seedling height was measured. $37 \mathrm{~d}$ after seedling establishment, root and tiller dry weights, and root to shoot ratios were determined. B. gracilis seedlings developed more tillers per seedling and higher heights under field capacity conditions than under the simulated-rain regime $(P \leq 0.05)$. Conversely, E. curvula seedlings grown under the simulated-rain regime developed more tillers and leaves than their counterparts grown under field capacity conditions, and than B. gracilis grown under both watering regimes. The two grasses showed a $90 \%$ establishment under field capacity conditions, but in boer lovegrass grown under the simulated-rain regime this variable decreased $10 \%$. The evaluation scheme proposed in this work might be a reliable tool to analyze the establishment and development of grass seedlings growing under greenhouse conditions and water stress.

Index words: Bouteloua gracilis, Eragrostis curvula, drought stress, field capacity, greenhouse

\section{INTRODUCCIÓN}

El severo deterioro de los pastizales de Norteamérica destaca la importancia de recuperar la productividad de estas comunidades mediante prácticas de rehabilitación como la resiembra de pastizales, en la cual la elección de las especies a resembrar es crítica dado el costo relativamente alto y la baja probabilidad de éxito de esta práctica. Bouteloua gracilis (Kunth) Lag. ex Griffiths es un pasto perenne dominante en los pastizales semiáridos de Norteamérica donde es considerado una de las especies clímax y el pasto nativo más importante del pastizal semiárido de México (Jaramillo, 1986) y de las praderas bajas de EE. UU. (Wilson y Briske, 1979).

Debido al severo deterioro de los pastizales de Norteamérica y la importancia de llevar a cabo la reintroducción de B. gracilis en su ambiente nativo, los factores que afectan el establecimiento de este pasto han sido ampliamente estudiados. A pesar de su importancia económica, ecológica y de ser considerado el pasto más promisorio para la revegetación de los pastizales semiáridos, el zacate navajita azul no es considerado como una alternativa viable para 
la resiembra de pastizales debido a las dificultades para establecerse en su ambiente natural (Wilson y Briske, 1979), aunque Wilson et al. (1976) indican algunas de las características de tolerancia a la sequía en plantas adultas de este zacate.

El crecimiento de B. gracilis comienza con la aparición de las primeras lluvias, pero en las condiciones limitativas de agua que comúnmente prevalecen en las regiones semiáridas, las plántulas del zacate navajita azul se deshidratan debido a la gran elongación del entrenudo subcoleoptilar que contiene al meristemo apical generador de las raíces adventicias que sustentan la supervivencia de las plantas adultas; este alargamiento provoca que las raíces adventicias sean formadas cerca de la superficie del suelo donde las condiciones ambientales son desfavorables para el desarrollo de las plántulas.

La sequía puede afectar el desarrollo de raíces de las plántulas de B. gracilis al reducir el área de la hoja y dañar directamente el primordio de la raíz y otros tejidos de la corona, pero una vez establecido el pasto no tiene problemas de persistencia (Briske y Wilson, 1980). Ante los problemas que presenta esta especie para lograr un establecimiento exitoso en condiciones naturales, la alternativa común para lograr la rehabilitación de los pastizales ha sido la introducción de especies de pastos exóticos, principalmente africanos (Corbin y D’Antonio, 2004; Ferrero-Serrano et al., 2011). Estas especies tienen importantes características morfológicas y de asignación de biomasa a raíces y hojas (Arredondo et al., 1998; Reich et al., 2001; McKing y Willson, 2006) que favorecen su éxito como invasoras.

Aunque el establecimiento y la producción de estas especies exóticas son comúnmente más altos que los de las especies nativas, el forraje producido por las primeras puede ser de una calidad relativamente menor (Jurado et al., 1988; Com. personal ${ }^{1}$ ). Además, las especies exóticas pueden desplazar a las especies nativas y reducir así la diversidad y riqueza de las comunidades nativas, además de alterar los pozos y flujos de energía y nutrientes de los ecosistemas (O’Connor, 1991; Saucedo-Monarque et al., 1997; Christian y Wilson, 1999). Una de las especies exóticas más utilizadas para la resiembra de pastizales es el pasto boer, Eragrostis curvula var. conferta, especie nativa de África que fue introducida a los Estados Unidos en 1932. Es una planta forrajera de tipo $\mathrm{C}_{4}$, perenne, resistente a la sequía pero no al frío, que se adapta bien a las áreas semidesérticas del suroeste de los Estados Unidos (Alderson y Sharp, 1993).

${ }^{1} J u r a d o$ G P, R L F Negrete, C R A Giner (1988) Resiembra comercial de dos zacates nativos y dos introducidos en los Altos de Jalisco: In: Resumen del $10^{\circ}$ Aniversario de Investigación del CIPEJ (1978-1988). Guadalajara, Jal. $14 \mathrm{p}$.
Ante la importancia de realizar la elección más adecuada de la especie a utilizar en los programas de rehabilitación de los pastizales semiáridos de Norteamérica, es importante diseñar esquemas de análisis en condiciones de invernadero que permitan evaluar el comportamiento y establecimiento de las especies a resembrar, para anticipar las probabilidades de éxito de esta práctica en campo. Los esquemas actuales de evaluación de establecimiento de plántulas consideran diseños experimentales más bien artificiales que no consideran la gran variabilidad del agua, el factor ambiental más importante para el crecimiento y desarrollo de las plantas en las zonas áridas y semiáridas (Aguado-Santacruz y García-Moya, 1998).

Por ejemplo, en algunos estudios se ha aplicado una primera fase de riego a capacidad de campo para posteriormente suspender el riego, y luego analizar la respuesta de las plántulas al déficit hídrico. Otros esquemas de estudio consideran periodos secuenciales de riego y sequía (Frasier et al., 1984; Esqueda et al., 2002), mientras que otros aplican un sistema de deshidratación gradual, en el cual la cantidad de agua para las plántulas se va reduciendo progresivamente con respecto a la capacidad de campo del sustrato o suelo empleado para la evaluación (Qi y Redman, 1993; Samarah, 2005).

Una alternativa a estos sistemas de evaluación es el riego por goteo o pulsado, en el que la frecuencia y la cantidad de agua aplicada determina la variación del potencial hídrico del suelo y, en consecuencia, la distribución de raíces y la captura de agua por la planta. Por lo general se aplican ciclos de descargas de agua de 1.6 a $8 \mathrm{~L} \mathrm{~h}^{-1}$ para mantener la humedad en el suelo por debajo de la capacidad de campo (Assouline et al., 2006; Bakeer et al., 2009; Ismail, 2010; Törnqvist y Jarsjö, 2012).

Ciertamente, el tipo de riego empleado afectará de manera fundamental la respuesta de las plantas a cualquier factor o tratamiento bajo análisis. Por ejemplo, comparado con sistemas de riego por goteo o pulsado, el riego a capacidad de campo puede ocasionar una lixiviación relativamente mayor de nutrientes hacia capas más profundas, ubicándolos por debajo del alcance de la raíz (Mmolawa y Or, 2000, Raine et al., 2007; Elmaloglou y Diamantopolous, 2007; Sezen et al., 2008; Sezen et al., 2010). Además, en suelos con alto contenido de arcilla este tipo de riego puede reducir el contenido de oxígeno disponible (Meyer y Barrs, 1991).

En el presente trabajo se evalúa un sistema generado a partir de datos climáticos reales para evaluar el establecimiento de plántulas. Al considerar la variabilidad de la lluvia, este esquema de análisis permite una mayor aproximación a las condiciones ambientales de establecimiento de las plantas en su ambiente nativo. Con los registros de lluvia y 
las dimensiones de los sistemas de captación de lluvia de la estación climática y de los contenedores utilizados para la evaluación del establecimiento de las plántulas, se calculan los volúmenes equivalentes de agua a aplicar diariamente en cada contenedor.

Si bien el sistema propuesto es laborioso y no logra la reproducción exacta de las condiciones ambientales que prevalecen en campo en una localidad y un periodo determinado, se apega más a la realidad que los sistemas de evaluación actuales y reproduce de manera más confiable la respuesta de las plántulas a la variabilidad en la disponibilidad de agua que caracteriza a los ambientes semiáridos, por lo que podría constituir un primer acercamiento a la predicción de las probabilidades de éxito de una resiembra. En el presente estudio este sistema de evaluación se empleó para evaluar el establecimiento y crecimiento de dos pastos forrajeros importantes para el país, Bouteloua gracilis, una especie nativa, y Eragrostis curvula var. conferta, una especie introducida.

\section{MATERIALES Y MÉTODOS}

\section{Colecta de material vegetal y manejo experimental}

Durante septiembre y octubre del 2010 se colectaron espigas de $B$. gracilis y E. curvula en poblaciones localizadas en 'Vaquerías', Mpo. de Ojuelos ( $21^{\circ} 46^{\prime}$ LN; $101^{\circ} 40^{\prime}$ LO), al noreste de Jalisco, México. La población nativa muestreada de $B$. gracilis fue tipificada molecularmente en un estudio previo (Aguado-Santacruz et al., 2004). Las cariópsides de los dos pastos fueron extraídas y seleccionadas cuidadosamente bajo un estereomicroscopio, para evitar material dañado o anormal, y se guardaron a $20^{\circ} \mathrm{C}$ para su uso posterior. Las cariópsides fueron esterilizadas con etanol $70 \%$ por 5 min y bicloruro de mercurio $\left(\mathrm{HgCl}_{2}\right) 0.02 \%$ por 30 min, y los restos de estos compuestos se eliminaron mediante tres lavados con agua destilada estéril.

La siembra de cariópsides para las evaluaciones fisiológicas de invernadero se hizo en contenedores de cartón de 7 x 7 x $19.5 \mathrm{~cm}$, perforados en su base. Estos contenedores se llenaron con $1235 \mathrm{~g}$ de suelo arcilloso arenoso estéril, cuyas características químicas se describen en el Cuadro 1 . Se ha comprobado que la textura arcillosa arenosa en el suelo es adecuada para la evaluación de la emergencia, crecimiento y supervivencia del zacate navajita azul (Stubbendieck et al., 1997; Esqueda et al., 2002).
En cada contenedor se sembraron cuatro cariópsides de B. gracilis o E. curvula a una profundidad de $1 \mathrm{~cm}$ (Carren et al., 1987), y ambas especies se sometieron a dos regímenes de riego: capacidad de campo (CC) y régimen simulado de lluvia (RS). La capacidad de campo para este suelo fue de $239 \mathrm{~mL}$ de agua por contenedor, determinada con la metodología descrita en Daubenmire (1974). Los contenedores a capacidad de campo se pesaron cada 2 d y se agregó el agua perdida por evapotranspiración para recuperar la capacidad de campo.

Otra parte de los contenedores se regó conforme a un régimen simulado de lluvia, generado a partir de registros climáticos reales obtenidos de una estación climática cercana al área de colecta de las semillas de B. gracilis y E. curvula, en 'Vaquerías', Jalisco. Para generar este régimen se calculó el volumen diario de agua captado a través del embudo del pluviómetro (área colectora $=401.15 \mathrm{~cm}^{2}$ ) durante un periodo de $37 \mathrm{~d}$ ( 1 junio al 7 de julio de 1987). Después, se determinó el volumen de agua correspondiente $(1 \mathrm{~mm}=4.7$ $\mathrm{mL}$; Cuadro 2) para las dimensiones de los contenedores (área $=49 \mathrm{~cm}^{2}$ ). Para simular el rocío matutino, diariamente se asperjó $1 \mathrm{~mL}$ de agua a la superficie del suelo de cada contenedor.

Estos registros climáticos se usaron debido a que las condiciones ambientales que prevalecieron durante ese año en 'Vaquerías' fueron favorables para el establecimiento exitoso de E. curvula durante una resiembra experimental realizada en el área (Jurado et al., 1988; Com. personal ${ }^{1}$ ). Ocho días después de la siembra se hizo un aclareo para uniformar el tamaño de las plántulas y dejar una por contenedor (12 contenedores o repeticiones por tratamiento). Antes del aclareo y hasta el día 8, los contenedores del régimen simulado de lluvia recibieron cuatro veces el volumen de agua indicado en el Cuadro 2, para posteriormente proporcionar las cantidades precisas de agua descritas para este régimen de riego.

Después del aclareo (día cero), cada $3 \mathrm{~d}$ se evaluaron las variables: altura de plántula, número de vástagos y número de hojas por plántula. Al final del experimento se calculó el porcentaje de establecimiento al dividir el número de plántulas al día cero entre el número de plántulas sobrevivientes al día 37, y multiplicar por 100. Posteriormente las plántulas fueron extraídas de los contenedores y separadas en raíces y vástagos. Este material se secó en un horno a $70{ }^{\circ} \mathrm{C}$ por $2 \mathrm{~d}$ para determinar los pesos secos, a partir de

Cuadro 1. Análisis elemental $\left(\mu \mathrm{g} \mathrm{g}^{-1}=\mathrm{ppm}\right)$ y contenido de materia orgánica $(\mathrm{MO}$, en \%) del suelo arcillosoarenoso usado en los experimentos de invernadero.

\begin{tabular}{ccccccccccc}
\hline $\mathrm{MO}$ & N-Inorg & P-Bray & $\mathrm{K}$ & $\mathrm{Ca}$ & $\mathrm{Mg}$ & $\mathrm{Na}$ & $\mathrm{Fe}$ & $\mathrm{Zn}$ & $\mathrm{Mn}$ & $\mathrm{Cu}$ \\
\hline 0.06 & 9.40 & 17.7 & 408 & 1680 & 286 & 222 & 2.96 & 0.33 & 4.16 & 0.45 \\
\hline
\end{tabular}


Cuadro 2. Régimen simulado de lluvia utilizado para evaluar el establecimiento de plántulas de Bouteloua gracilis y Eragrostis curvula var. conferta bajo condiciones de invernadero.

\begin{tabular}{|c|c|c|c|}
\hline $\begin{array}{l}\text { Días consecutivos del régimen } \\
\text { simulado de lluvia }\end{array}$ & $\begin{array}{l}\text { Fecha de registro de } \\
\text { la precipitación }\end{array}$ & $\begin{array}{l}\text { Precipitación registrada } \\
\qquad(\mathrm{mm})\end{array}$ & $\begin{array}{l}\text { Riego equivalente } \\
(\mathrm{mL})^{\dagger \dagger}\end{array}$ \\
\hline 1 & Junio 1 & 9.8 & 46.1 \\
\hline 2 & Junio 2 & 4.6 & 21.6 \\
\hline 3 & Junio 3 & 0 & 0.0 \\
\hline 4 & Junio 4 & 0 & 0.0 \\
\hline 5 & Junio 5 & 17.7 & 83.2 \\
\hline 6 & Junio 6 & 1 & 4.7 \\
\hline 7 & Junio 7 & 1 & 4.7 \\
\hline 8 & Junio 8 & 0 & 0.0 \\
\hline $9^{\dagger}$ & Junio 9 & 0 & 0.0 \\
\hline 10 & Junio 10 & 3.2 & 15.0 \\
\hline 11 & Junio 11 & 0 & 0.0 \\
\hline 12 & Junio 12 & 0 & 0.0 \\
\hline 13 & Junio 13 & 0 & 0.0 \\
\hline 14 & Junio 14 & 0 & 0.0 \\
\hline 15 & Junio 15 & 15.3 & 71.9 \\
\hline 16 & Junio 16 & 0 & 0.0 \\
\hline 17 & Junio 17 & 0 & 0.0 \\
\hline 18 & Junio 18 & 0 & 0.0 \\
\hline 19 & Junio 19 & 0 & 0.0 \\
\hline 20 & Junio 20 & 0 & 0.0 \\
\hline 21 & Junio 21 & 0 & 0.0 \\
\hline 22 & Junio 22 & 0 & 0.0 \\
\hline 23 & Junio 23 & 1 & 4.7 \\
\hline 24 & Junio 24 & 0 & 0.0 \\
\hline 25 & Junio 25 & 0 & 0.0 \\
\hline 26 & Junio 26 & 0 & 0.0 \\
\hline 27 & Junio 27 & 0 & 0.0 \\
\hline 28 & Junio 28 & 0 & 0.0 \\
\hline 29 & Junio 29 & 0 & 0.0 \\
\hline 30 & Junio 30 & 1.5 & 7.1 \\
\hline 31 & Julio 1 & 0 & 0.0 \\
\hline 32 & Julio 2 & 4 & 18.8 \\
\hline 33 & Julio 3 & 0 & 0.0 \\
\hline 34 & Julio 4 & 0 & 0.0 \\
\hline 35 & Julio 5 & 1 & 4.7 \\
\hline 36 & Julio 6 & 0 & 0.0 \\
\hline 37 & Julio 7 & 0 & 0.0 \\
\hline Total & & 60.1 & 282.5 \\
\hline
\end{tabular}

${ }^{\dagger}$ Día en que se hizo el aclareo a una plántula por contenedor (día 0). ${ }^{\dagger \dagger}$ Capacidad de campo $=239 \mathrm{~mL}$ 
los cuales se calcularon las relaciones raíz/vástago. Durante el experimento, la temperatura promedio en el invernadero fue de $28.9^{\circ} \mathrm{C}$.

\section{Análisis estadístico}

Los datos se sometieron a un análisis completamente al azar, y cuando se detectaron valores significativos de $\mathrm{F}$, se usó la prueba de Tukey $(\alpha=0.05)$ para la comparación de medias (Steel y Torrie, 1988). Para el análisis estadístico del porcentaje de establecimiento, los datos fueron sujetos a una transformación angular de arco seno $(\mathrm{P} \leq 0.05$; Steel y Torrie, 1988).

\section{RESULTADOS Y DISCUSIÓN}

Los dos regímenes de riego afectaron el desarrollo de las plántulas de Bouteloua gracilis y Eragrostis curvula. Con el régimen simulado de lluvia nunca se alcanzaron las condiciones de capacidad de campo, y el periodo ubicado entre los días 16 al 37 fue el más crítico para el crecimiento de las plántulas, ya que después del último riego relativamente abundante aplicado el día $15(71.9 \mathrm{~mL}$, equivalente a $30 \%$ de la CC), sólo se aplicaron tres riegos adicionales de menor magnitud: $4.7 \mathrm{~mL}$ los días 23 y 35 ( $2 \%$ de la CC), $7.1 \mathrm{~mL}$ el día 30 (3\% de la CC) y $18.8 \mathrm{~mL}$ el día 32 (8\% de la CC).

\section{Crecimiento de las plántulas}

Las plántulas de E. curvula y B. gracilis crecidas bajo el régimen simulado de lluvia mostraron, en términos generales, mayor altura que sus contrapartes crecidas con riegos a capacidad de campo, por lo menos hasta el día 31 (Figura 1a). Posteriormente, cuando la escasez de agua se hizo crítica esta situación se invirtió, de modo que las plantas crecidas a capacidad de campo comenzaran a desarrollar mayor altura que las plántulas crecidas bajo el régimen simulado de lluvia. Al final del experimento, las plántulas de B. gracilis crecidas a capacidad de campo alcanzaron la mayor altura, ( $\mathrm{P} \leq 0.05$; Cuadro 3$)$ mientras que en E. curvula la diferencia no fue significativa.

En cambio, bajo el régimen simulado de lluvia las plántulas de E. curvula desarrollaron de manera consistente durante casi todo el periodo experimental, una mayor cantidad de vástagos secundarios que las de $B$. gracilis y que sus propias plántulas crecidas a capacidad de campo (Figura 1b); no obstante, al final las plántulas de E. curvula tuvieron la misma cantidad de vástagos secundarios con el régimen simulado de lluvia que con riego a capacidad de campo (1.3 $v s .1 .4$ vástagos por plántula). Las plántulas de ambos pastos formaron la misma cantidad de vástagos bajo condiciones de capacidad de campo, mientras que $B$. gracilis formó mayor cantidad de vástagos bajo condiciones de capacidad de campo que bajo el régimen simulado de lluvia $(\mathrm{P} \leq 0.05$; Cuadro 3).

La formación de hojas por plántula también fue mayor en E. curvula hasta el día 31 (Figura 1c). Posteriormente, el número de hojas por plántula tanto en E. curvula como en $B$. gracilis fue afectado por el déficit de agua, de modo que al final del experimento las plántulas de ambos pastos regadas a capacidad de campo produjeron mayor cantidad de hojas que sus contrapartes crecidas bajo el régimen simulado de lluvia ( $\mathrm{P} \leq 0.05$; Cuadro 3 ). Sin embargo, el número de hojas entre los dos pastos crecidos en condiciones de capacidad de campo o riego simulado no fue estadísticamente diferente al final del experimento ( $\mathrm{P}>0.05$; Cuadro 3$)$.

Los resultados anteriores muestran que el efecto de la escasez de agua en el régimen simulado de lluvia se hizo evidente hasta después del día 31, cuando decreció el número de hojas en las plántulas. Estos datos también muestran que en ambas especies de pastos la formación de hojas resultó ser más sensible al déficit hídrico que la formación de vástagos. Además, fue evidente que bajo condiciones de restricción de humedad, E. curvula se desarrolló mejor que $B$. gracilis, al menos en cuanto a la formación de hojas (Figura 1c) y vástagos (Figura $1 b)$.

Köchy y Wilson (2004) encontraron que para B. gracilis la densidad de vástagos fue más alta en plantas que recibieron mayor cantidad de agua que en las que recibieron poca; similarmente, Briske y Wilson (1980) encontraron una relación positiva entre el potencial hídrico del suelo y la formación de vástagos en esta misma especie. Al respecto, Wilson y Sarles (1978) y Emam et al. (2010) señalaron que el ajuste del área foliar constituye un proceso importante para reducir la pérdida de agua a través de la disminución del área transpiratoria, ya sea mediante la pérdida de hojas o la disminución del crecimiento foliar.

También Majerus (1975) encontró una clara relación entre potencial hídrico del suelo y el crecimiento de hojas en el pasto $B$. gracilis, ya que una vez que el potencial hídrico del suelo a $25 \mathrm{~cm}$ de profundidad fue menor de -1 bar, el crecimiento de hojas se redujo en cerca de $50 \%$. La producción de hojas se ha considerado un proceso íntimamente ligado a la formación de vástagos (Aguado-Santacruz et al., 2009). E. curvula formó mayor número de hojas que el zacate navajita azul bajo el régimen simulado de lluvia, lo cual coincide con los resultados reportados por Carrillo et al. (2009) quienes observaron una eficiencia superior a 90 $\%$ en la producción de hojas en la especie exótica Eragrostis lehmanniana en comparación con la especie nativa $B$. gracilis, mientras que Arredondo et al. (1998) concluyeron que las especies invasoras pueden producir mayor área foliar que las especies nativas. 

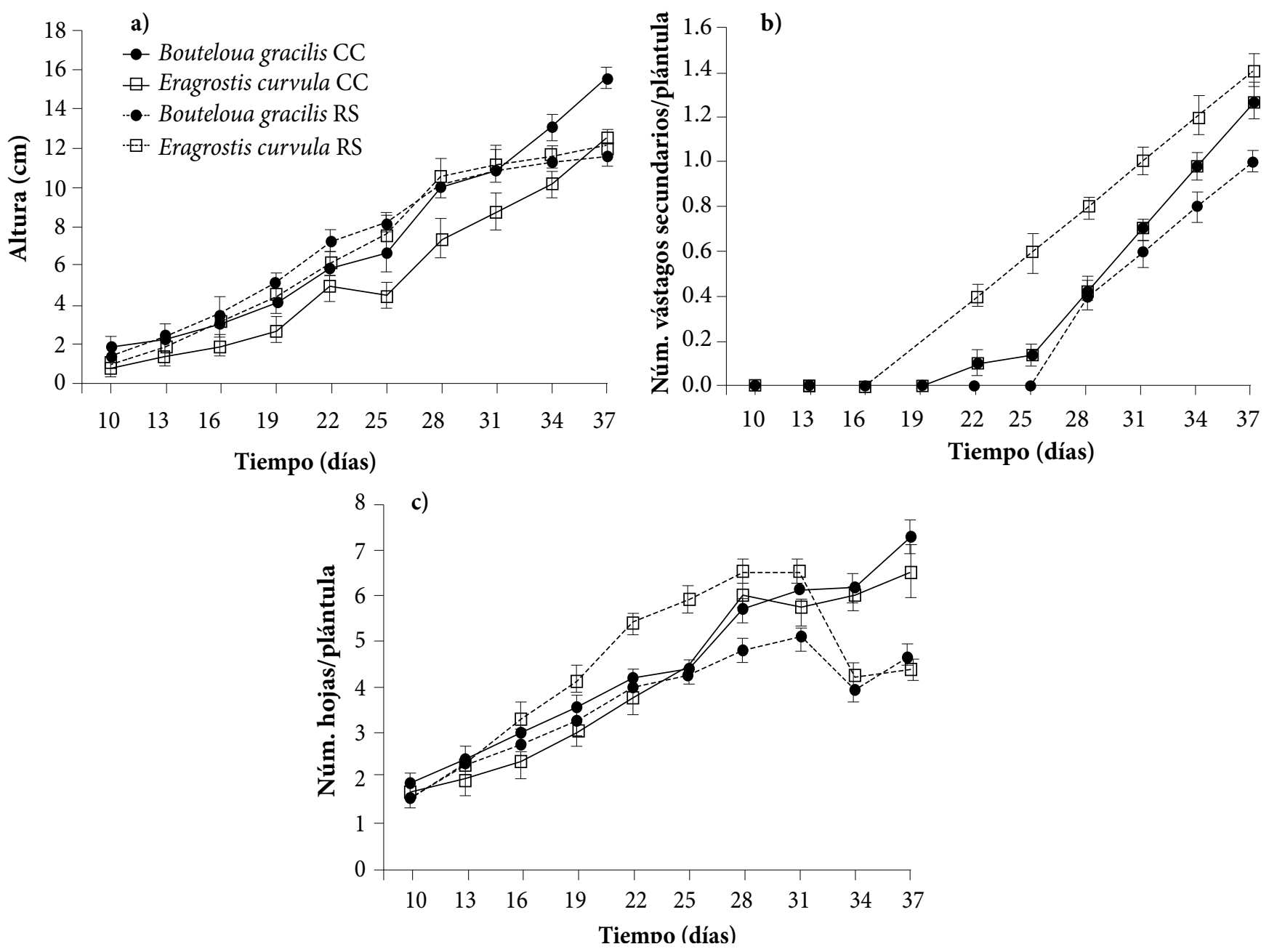

Figura 1. Altura de plántula (a), número de vástagos secundarios por plántula (b) y número de hojas por plántula (c) en Bouteloua gracilis y Eragrostis curvula var. conferta crecidas bajo dos regímenes de riego $(\mathrm{CC}=$ capacidad de campo y $\mathrm{RS}=$ régimen simulado de lluvia). Las barras verticales representan el error estándar.

Cuadro 3. Crecimiento de plántulas de Bouteloua gracilis y Eragrostis curvula var. conferta bajo dos regímenes de humedad, al final del experimento ( $37 \mathrm{~d})$.

\begin{tabular}{|c|c|c|c|c|}
\hline \multirow{3}{*}{ Variable } & \multicolumn{4}{|c|}{ Especie } \\
\hline & \multicolumn{2}{|c|}{ Bouteloua gracilis } & \multicolumn{2}{|c|}{ Eragrostis curvula } \\
\hline & $\mathrm{CC}$ & RS & $\mathrm{CC}$ & RS \\
\hline Altura de plántula $(\mathrm{cm})$ & $15.6 \mathrm{a}$ & $11.6 \mathrm{~b}$ & $12.5 \mathrm{~b}$ & $12.1 \mathrm{~b}$ \\
\hline Núm.de vástagos & $1.3 \mathrm{a}$ & $1.0 \mathrm{~b}$ & $1.3 \mathrm{a}$ & $1.4 \mathrm{a}$ \\
\hline Núm. de hojas & $7.3 \mathrm{a}$ & $4.6 \mathrm{~b}$ & $6.5 \mathrm{a}$ & $4.4 \mathrm{~b}$ \\
\hline
\end{tabular}

Medias con literales distintas en cada hilera indican diferencias significativas entre especies y regímenes de riego (Tukey, 0.05). CC = capacidad de campo; RS = régimen simulado de lluvia.

\section{Biomasa de raíces y vástagos}

Los pesos secos de vástago y raíz al final del experimento fueron mayores en E. curvula que en B. gracilis, bajo las dos condiciones de riego $(\mathrm{P} \leq 0.05$; Figura 2$)$. En ambos pastos la acumulación de biomasa en raíces y vástagos fue menor bajo el régimen simulado de lluvia que bajo capacidad de campo. E. curvula produjo raíces más largas que B. gracilis bajo cualquier condición de riego, y en ambos pastos las raíces fueron de menor longitud bajo el régimen simulado de lluvia que bajo condiciones de capacidad de campo (Figura 3). 
Algunos investigadores (Huang y Fry, 1998; Huang y Gao, 2000; Thorne y Frank, 2009) han demostrado que el estrés hídrico provoca un aumento en la longitud de las raíces, mientras que otros (Mohammad et al., 1982; Fresnillo-Fedorenko et al., 1995; Busso y Bolletta, 2007; Ervin et al., 2009) encontraron que el crecimiento de la raíz en algunas especies de pastos perennes puede ser menor bajo condiciones limitativas de agua, lo cual representaría una ventaja en condiciones naturales ya que un sistema radical superficial permitiría aprovechar mejor el agua cuando la cantidad de lluvia es baja $(<5 \mathrm{~mm}$; Sala y Laurenroth, 1982).

Puesto que en el presente estudio ambos pastos desa- rrollaron una mayor longitud de raíz bajo condiciones de mayor disponibilidad de agua, es posible que estas especies tengan raíces más cortas cuando hay escases de agua, como estrategia de supervivencia. Alternativamente, es probable que la cantidad de agua suministrada durante este estudio haya sido adecuada para el desarrollo de ambos pastos durante los primeros $31 \mathrm{~d}$ y sólo hasta después la falta de agua afectó el crecimiento de las plántulas. Si se considera que el requerimiento óptimo de agua de E. curvula es de $33 \mathrm{~mm}$ de precipitación al año en condiciones naturales (Halvorson y Guertin, 2003) y que el régimen simulado de lluvia aportó un total de $60.1 \mathrm{~mm}$ (Cuadro 2), entonces se podría inferir que el régimen de riego aplicado no representó en realidad una situación de estrés para E. curvula.

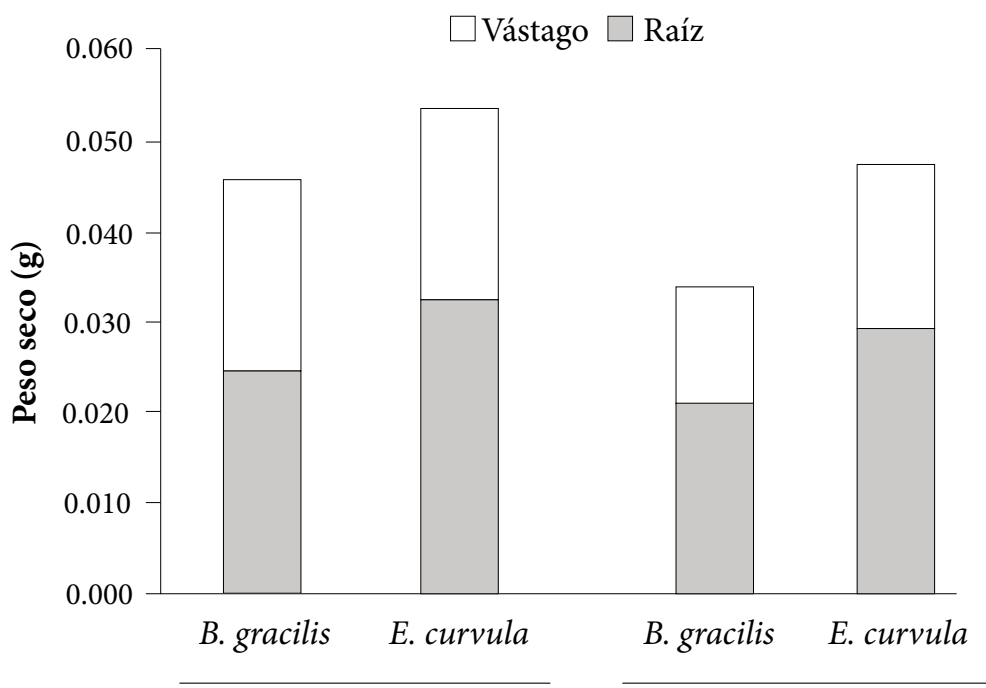

Riego a capacidad de campo Régimen simulado de lluvia

Figura 2. Peso seco de raíz y vástago de plántulas de Bouteloua gracilis y Eragrostis curvula var. conferta crecidas bajo dos regímenes de riego. Letras distintas indican diferencias significativas (Tukey, 0.05).
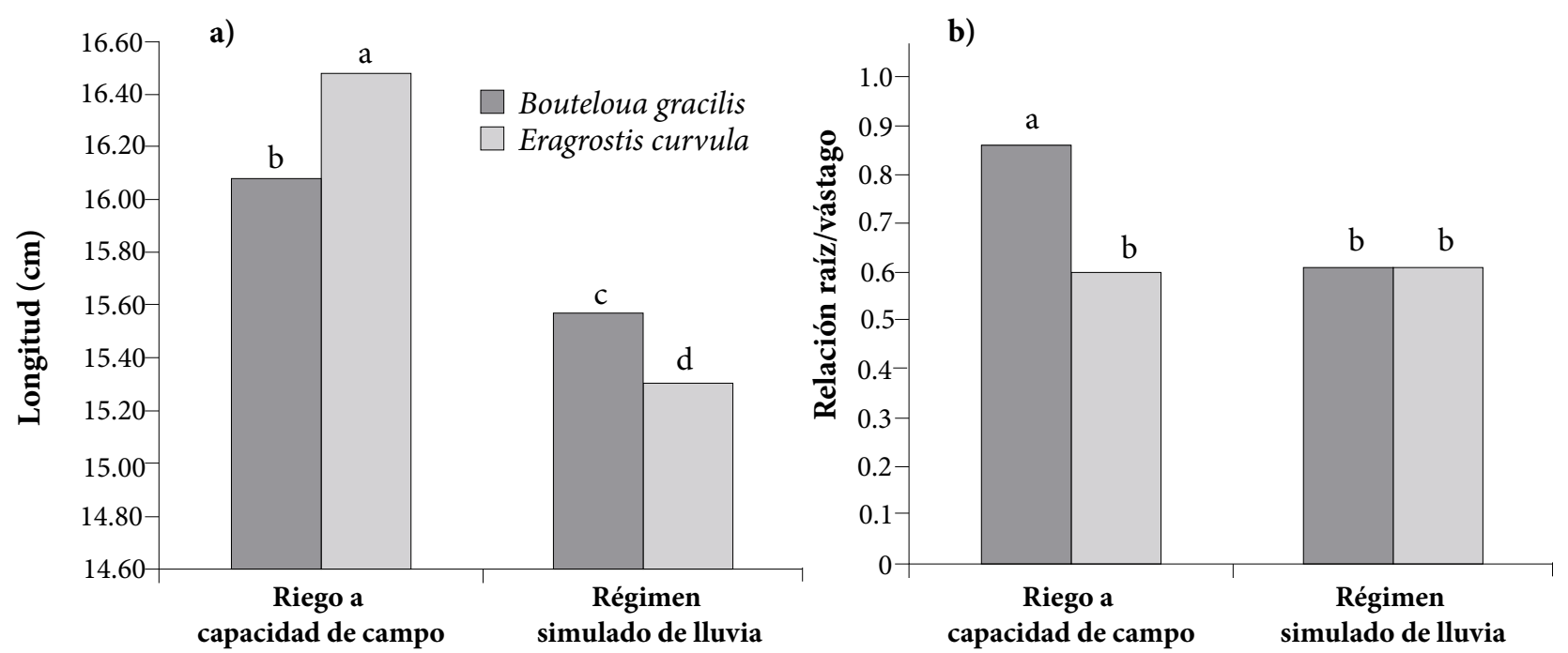

Figura 3. Longitud de raíz (a) y relación raíz/vástago (b) en plántulas de Bouteloua gracilis y Eragrostis curvula var. conferta crecidas bajo dos regímenes de riego. Letras distintas en cada gráfica indican diferencias significativas (Tukey, 0.05). 
El tipo y la frecuencia del riego son factores que afectan el comportamiento fisiológico y productivo de las plantas (Assouline et al., 2006; Méndez-Natera et al., 2007; Tapia-Vargas et al., 2010). Assouline et al. (2006) y El Saidi et al. (2010) han estudiado el riego pulsado por goteo, el cual se aplica generalmente entre 1.6 a $8 \mathrm{~L} \mathrm{~h}^{-1}$, con intervalos cortos sin riego. En estos sistemas, la cantidad de agua aplicada es optimizada en comparación con otros en los que el agua suministrada es demasiada, ya que la raíz no es capaz de aprovecharla o almacenarla en su totalidad, por lo que ocurre un lavado de nutrientes del suelo.

Según Assouline et al. (2006), el aumento de la frecuencia de riegos cortos resulta en un incremento de la disponibilidad de agua y nutrientes en la zona activa de la raíz, contrario a lo que sucede con los riegos frecuentes a capacidad de campo. Es decir, las condiciones de capacidad de campo usadas en este estudio pudieron ocasionar que los nutrientes se filtraran a zonas más profundas de las macetas, fuera del alcance de las raíces relativamente superficiales de las plántulas evaluadas.

El zacate navajita azul presentó una relación raíz/vástago mayor bajo condiciones de capacidad de campo $(\mathrm{P}<0.05)$, mientras que esta variable fue igual entre las dos condiciones de riego para E. curvula (Figura 3). La menor relación raíz/vástago presentada por $B$. gracilis bajo el régimen simulado de lluvia en comparación con capacidad de campo, coincide con lo reportado por Baruch y Jackson (2005) quienes observaron que el estrés hídrico redujo las relaciones raíz/vástago en varias especies de pastos (nativos e invasores $\mathrm{C}_{4}$ ).

En cambio, Bakker y Wilson (2001) encontraron que la disponibilidad de agua no tuvo efecto en la supervivencia, índice de crecimiento, relación raíz/vástago o en la distribución de biomasa de B. gracilis, y concluyeron que el crecimiento de plantas individuales de $B$. gracilis no parece estar limitado por la disponibilidad de agua. En E. curvula se ha encontrado que su parte aérea crece a menor tasa que en las especies nativas (Cox y Martin, 1984); esta característica de baja biomasa aérea reduce su tasa de transpiración y sus requerimientos nutricionales, por lo que requiere un menor consumo de agua para sobrevivir. Esta y otras características hacen de E. curvula una especie resistente a condiciones de estrés de humedad (Frasier et al., 1985).

\section{Establecimiento de las plántulas}

Es conocido que el zacate navajita azul presenta dificultades para establecerse bajo condiciones naturales, por lo que sus requerimientos de establecimiento han sido bien estudiados. Según Wilson y Briske (1979), el zacate navajita azul requiere de 2 a $4 \mathrm{~d}$ de condiciones de humedad en el suelo para germinar e iniciar el crecimiento de la raíz seminal; en las siguientes 2 a 8 semanas requiere una condición similar de humedad para que haya crecimiento de las raíces adventicias y se asegure el establecimiento definitivo de las plántulas.

En el presente estudio estos dos periodos de humedad fueron satisfechos adecuadamente con dos riegos importantes aplicados durante los primeros días posteriores a la siembra (46.1 mL el día 1 y $83.2 \mathrm{~mL}$ el día 5) y dos riegos significativos $14 \mathrm{~d}$ después de la siembra $(71.9 \mathrm{~mL}$ el día 15 y $18.8 \mathrm{~mL}$ el día 32; Cuadro 2). Esqueda et al. (2005) demostraron que la germinación inicial y la supervivencia de plántulas de esta gramínea están directamente relacionadas con los periodos de humedad y sequía después de la siembra.

Bajo las condiciones experimentales del presente estudio, ambos pastos lograron altos porcentajes de establecimiento tanto en condiciones de capacidad de campo como en el régimen simulado de lluvia. Contrario a lo que se esperaba, E. curvula presentó una disminución de 90 a $80 \%$ en su establecimiento bajo el régimen simulado de lluvia, mientras que B. gracilis lo mantuvo en 90 \% (Figura 4).

Se ha reportado que cuando existe un exceso de humedad baja el establecimiento de las plántulas de E. curvula debido a infecciones por hongos y nematodos, así como por competencia con otras plantas (Cox et al., 1987). En respuesta a la sequía las raíces adventicias de las plántulas deben desarrollarse lo suficiente para asegurar que las hojas y tallos reciban suficiente agua y nutrientes antes de que las plántulas se consideren establecidas (Ries y Svejcar, 1991). En este estudio, las plantas de E. curvula sometidas al régimen simulado de lluvia fueron las que presentaron menor longitud de raíz, hecho que pudo afectar su establecimiento.

En lo que respecta a $B$. gracilis, algunos autores señalan que las plántulas se consideran establecidas cuando han formado seis hojas, dos raíces adventicias que penetran 10 $\mathrm{cm}$, y dos vástagos secundarios por planta (Ries y Svejcar, 1991). Aquí se encontró que las plántulas de zacate navajita azul alcanzaron más de $15 \mathrm{~cm}$ de longitud en ambos regímenes de riego, por lo que se consideraron establecidas a los 37 d después de la siembra. Estos datos coinciden con Ries y Svejcar (1991), quienes estimaron un periodo similar (46 d) para el establecimiento de plántulas de esta especie en campo; a los 39 d de su experimento, el número de vástagos y hojas fueron muy similares a los aquí obtenidos al final del estudio. 


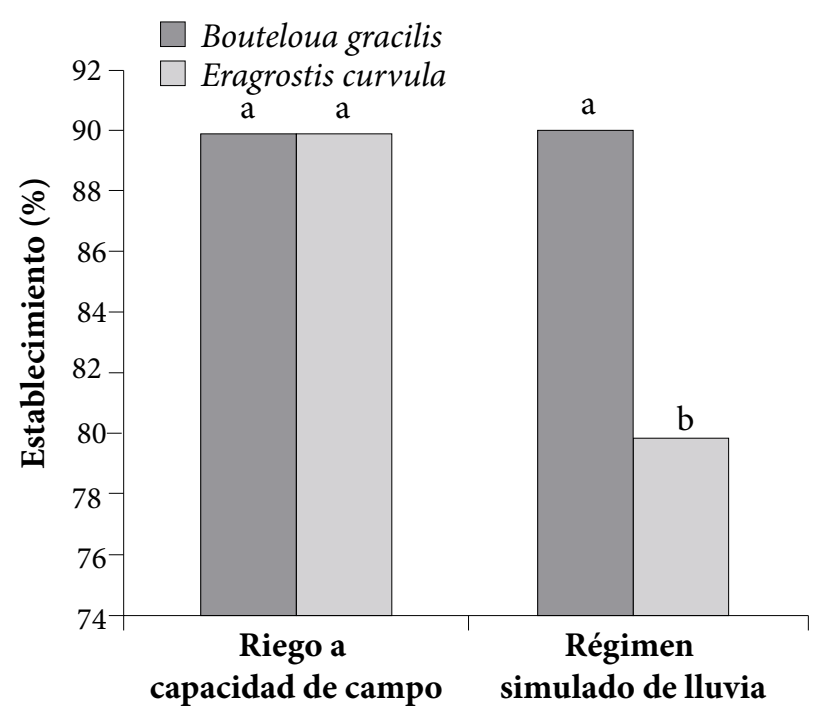

Figura 4. Establecimiento de Bouteloua gracilis y Eragrostis curvula var. conferta bajo dos regímenes de riego. Letras distintas indican diferencias significativas (Tukey, 0.05).

\section{CONCLUSIONES}

Aunque el régimen simulado de lluvia utilizado en este trabajo para evaluar el establecimiento de plántulas de pastos en invernadero, no logra una reproducción exacta de las condiciones de campo, tal método puede ser una herramienta más confiable que otros métodos, para anticipar los probables resultados de una resiembra en campo. Los resultados aquí obtenidos con el régimen simulado de lluvia confirman hallazgos previos reportados por otros autores, particularmente la tolerancia relativa de E. curvula a la deficiencia de agua en el suelo, y la necesidad de $B$. gracilis de disponer de mejores condiciones de humedad para lograr un adecuado crecimiento y establecimiento de plántulas. También se evidencia que la formación de hojas es un proceso que se ve afectado en mayor grado por las condiciones de estrés hídrico que la formación de vástagos, particularmente en E. curvula.

\section{BIBLIOGRAFÍA}

Aguado-Santacruz G A, E García-Moya (1998) Environmental factors and community dynamics at the southernmost part of the North American Graminetum. Plant Ecol. 135:13-29.

Aguado-Santacruz G A, N E Leyva-López, K I Pérez-Márquez, E García-Moya, J T Arredondo-Moreno, J P Martínez-Soriano (2004) Genetic variability of Bouteloua gracilis populations differing in forage production at the southernmost part of the North American Graminetum. Plant Ecol. 170:287-299.

Aguado-Santacruz G A, Q Rascón-Cruz, B Moreno-Gómez, R G Guevara-González, L Guevara-Olvera, J F Jiménez-Bremont, S Arévalo-Gallegos, E García-Moya (2009) Genetic transformation of blue grama grass with the rol Agene from Agrobacterium rhizogenes: regeneration of transgenic plants involves a "hairy embryo" stage. In Vitro Cell. Develop. Biol.-Plant 45:681-692.

Alderson J, W C Sharp (1993) Grass Varieties in the United States. Agricultural Handbook No. 170. United States Department of Agriculture, Washington, DC. $72 \mathrm{p}$.
Arredondo J T, T A Jones, D A Johnson (1998) Seedling growth of intermountain perennial and weedy annual grasses. J. Range Manage. 51:584-589.

Assouline S, M Möller, S Cohen, M Ben-Hur, A Grava, K Narkis, A Silber (2006) Soil plant system response to pulsed drip irrigation and salinity: Bell pepper case study. Soil Sci. Soc. Amer. J. 70:1556-1568.

Bakker J, S Wilson (2001) Competitive abilities of introduced and native grasses. Plant Ecol. 157:117-125.

Bakeer G A A, F G El-Ebabi, M T El-Saidi (2009) Effect of pulse drip irrigation on yield and water use efficiency of potato crop under organic agriculture in sandy soils. Misr J. Agric. Eng. 26:736765.

Baruch Z, R B Jackson (2005) Responses of tropical native and invader $\mathrm{C} 4$ grasses to water stress, clipping and increased atmospheric CO2 concentration. Oecologia 145:522-532.

Briske D D, A M Wilson (1980) Drought effects on adventitious root development in blue grama seedlings. J. Range Manage. 33:323327.

Busso C A, A I Bolletta (2007) Perennial grasses of different successional stages under various soil water inputs: Do they differ in root length density? Interciencia 32:205-212

Carren C J, A M Wilson, R L Cuany (1987) Caryopsis weight and planting depth of blue grama. II. Emergence in Marginal Soil Moisture. J. Range Manage. 40:212-216.

Carrillo S S, M T Arredondo, E Huber-Sannwald, R J Flores (2009) Comparación en la germinación de semillas y crecimiento de plántulas entre gramíneas nativas y exóticas del pastizal semiárido. Téc. Pecu. Méx. 47:299-312.

Christian J M, D S Wilson (1999) Long-term ecosystem impacts of an introduced grass in the northern Great Plains. Ecology 80:23972407.

Corbin J D, C M D'Antonio (2004) Competition between native perennial and exotic annual grasses: implications for an historical invasion. Ecology 85:1273-1283.

Cox J, M Martin (1984) Effects of planting depth and soil textures on the emergence of four lovegrasses. J. Range Manage. 37:453-458.

Cox J R, M H Martin-R, F A Ibarra-F, J H Fourie, M F G Rethman, D G Wilcox (1987) Effects of climate and soils on the distribution of four African grasses: In: Seed and Seedbed Ecology of Rangeland Plants: Proc. of Symposium. W G Frasier, Evans R A (eds). U.S. Department of Agriculture, Agricultural Research Service. Tucson, AZ. Washington, DC. pp:225-241. 
Daubenmire R F (1974) Plants and Environment. A Textbook of Autoecology. John Wiley and Sons Inc. New York. $422 \mathrm{p}$.

Elmaloglou S, E Diamantopoulos (2007) Wetting front advance patterns and water losses by deep percolation under the root zone as influenced by pulsed drip irrigation. Agric. Water Manage. 90:160-163.

El Saidi M T, O M Kassab, E M Okasha, A R E Abdelghany (2010) Effect of drip irrigation systems, water regimes and irrigation frequency on growth and quality of potato under organic agriculture in sandy soils. Aust. J. Basic Appl. Sci. 4:4131-4141.

Emam Y, A Shekoofa, F Salehi, A H Jalali (2010) Water stress effects on two common bean cultivars with contrasting growth habits. American-Eurasian J. Agric. Environ. Sci. 9:495-499.

Ervin E H, A LaBranche, X Zhang (2009) Kentucky bluegrass and creeping bentgrass responses to foliar application of glycinebetaine at three et replacement levels. Int. Turfgrass Soc. 11:755-763.

Esqueda C M H, C A Melgoza, C M Sosa, R R Carrillo, J Jiménez (2005) Emergencia y sobrevivencia de gramíneas con diferentes secuencias de humedad/sequía en tres tipos de suelo. Téc. Pecu. Méx. 43:101-115.

Esqueda C M H, R R L Carrillo, C M Sosa, C A Melgoza, M M H Royo, J Jiménez (2002) Emergencia y supervivencia de gramíneas inoculadas con biofertilizantes en condiciones de invernadero. Téc. Pecu. Méx. 42:459-475.

Ferrero-Serrano A, A L Hild, B A Mealor (2011) Can invasive species enhance competitive ability and restoration potential in native grass populations? Restor. Ecol. 19:545-551.

Frasier G W, D A Woolhiser, J R Cox (1984) Emergence and seedling survival of two warm-season grasses as influenced by the timing of precipitation: a greenhouse study. J. Range Manage. 37:7-11.

Frasier G W, J R Cox, D A Woolhiser (1985) Emergence and survival response of seven grasses for six wet-dry sequences. J. Range Manage. 38:372-377.

Fresnillo-Fedorenko D E, O A Fernández, C A Busso (1995) The effect of water stress on top and root growth in Medicago minima. J. Arid Environ. 29:47-54.

Halvorson W L, P Guertin (2003) Status of Introduced Plants in Southern Arizona Parks Factsheet for Eragrostis curvula (Schrad.) Nees and Eragrostis curvula var. conferta Stapf. U.S. Geological Survey/Southwest Biological Science Center University of Arizona. Tucson, Arizona. $36 \mathrm{p}$

Huang B, H Gao (2000) Root physiological characteristics associated with drought resistance in tall fescue cultivars. Crop Sci. 40:196-203.

Huang B, J Fry (1998) Root anatomical, physiological and morphological responses to drought stress for tall fescue cultivars. Crop Sci. 38:1017-1022.

Ismail S M (2010) Influence of deficit irrigation on water use efficiency and bird pepper production (Capsicum annuum L.). JKAU: Meteor. Environ. Arid Land Agric. Sci. 21:29-43.

Jaramillo V V (1986) La importancia de los coeficientes de agostadero y de las gramíneas en el manejo de los agostaderos del país: In: Mem. $2^{\circ}$ Congreso Nacional sobre Manejo de Pastizales. C J Gutiérrez (ed). Saltillo, Coah. pp:8-15.

Köchy M, S D Wilson (2004) Semiarid grassland responses to short-term variation in water availability. Plant Ecol. 174:197-203.

Majerus M E (1975) Response of root and shoot growth of 3 grass species to decreases in soil water potential. J. Range Manage. 28:473476.

McKing W, B J Wilson (2006) Differentiation between native and exotic plant species from a dry grassland: fundamental responses to resource availability, and growth rates. Austral Ecol. 31:9961004 .
Méndez-Natera J R, R S Salazar-Brito, J Merazo-Pinto, J A Gil-Marín, L Khan-Prado (2007) Efecto de tres frecuencias de riego sobre el rendimiento de algodón en rama y sus componentes en cuatro cultivares de algodón (Gossypium hirsutum L.) tipo upland. Rev. Fac. Agron. Univ. Zulia 24:229-256.

Meyer W S, H D Barrs (1991) Roots in irrigated clay soils: Measurement techniques and responses to rootzone conditions. Irrigation Sci. 12:125-134

Mmolawa K, D Or (2000) Root zone solute dynamics under drip irrigation: A review. Plant Soil 222:163-190.

Mohammad N, D D Dwyer, F E Busby (1982) Responses of crested wheatgrass and Russian wild rye to water stress and defoliation. J. Range Manage. 35:227-230.

O'Connor T G (1991) Local extinction in perennial grasslands: a life-history approach. Amer. Nat. 137:753-773.

Qi M O, R E Redman (1993) Seed germination and seedling survival of C3 and C4 grasses under water stress. J. Arid Environ. 24:277285.

Raine S R, W S Meyer, D W Rassam, J L Hutson, F J Cook (2007) Soil-water and solute movement under precision irrigation: knowledge gaps for managing sustainable root zones. Irrigation Sci. 26:91-100

Reich P B, D Tilman, J Craine, D Ellsworth, M G Tjoelker, J Knops (2001) Do species and functional groups differ in acquisition and use of $\mathrm{C}, \mathrm{N}$ and water under varying atmospheric $\mathrm{CO} 2$ and $\mathrm{N}$ availability regimes? A field test with 16 grassland species. New Phytol.150:435-448.

Ries R E, T J Svejcar (1991) The grass seedling: when is it established? J. Range Manage. 44:574-576.

Sala O E, W K Lauenroth (1982) Small rainfall events: an ecological role in semiarid regions. Oecologia 53:301-304.

Samarah N H (2005) Effects of drought stress on growth and yield of barley. Agron. Sust. Develop. 25:145-149

Saucedo-Monarque E, E García-Moya, J L Flores-Flores (1997) La riqueza, una variable de respuesta de la vegetación a la introducción del zacate buffel. Agrociencia 31:83-89.

Sezen S M, A Yazar, A Akyildiz, H Y Dasgan (2008) Yield and quality response of drip irrigated green beans under full and deficit irrigation. Sci. Hort. 117:95-102.

Sezen S M, G Celikel, A Yazar, S Tekin, B Kapur (2010) Effect of irrigation management on yield and quality of tomatoes grown in different soilless media in a glasshouse. Sci. Res. Essay 5:041-048.

Steel R G D, J H Torrie (1988) Bioestadística: Principios y Procedimientos. 2da. ed. McGraw Hill/Interamericana de México. 622 p.

Stubbendieck J, S L Hatch, C H Butterfield (1997) North American Range Plants. University of Nebraska Press. Lincoln, NE. 501 p.

Tapia-Vargas M, A Larios-Guzmán, I Abrisqueta-Villena, O Mounzer, J Vera-Muñoz, J M Abrisqueta-García, M C Ruiz Sánchez (2010) Riego deficitario en melocotonero. Análisis del rendimiento y de la eficiencia en el uso del agua. Rev. Fitotec. Mex. 33:89-93.

Thorne M A, D A Frank (2009) The effects of clipping and soil moisture on leaf and root morphology and root respiration in two temperate and two tropical grasses. Plant Ecol. 200:205-215.

Törnqvist R, J Jarsjö (2012) Water Savings through improved irrigation techniques: basin-scale quantification in semi-arid environments. Water Resour. Manage. 26:949-962.

Wilson A M, D N Hyder, D D Briske (1976) Drought resistance characteristics of blue gramma seedlings. Agron. J. 68:479-484.

Wilson A M, D D Briske (1979) Seminal and adventitious root growth of blue gramma seedlings on the Central Plains. J. Range Manage. 32:209-213.

Wilson A M, J A Sarles (1978) Quantification of drought tolerance and avoidance of blue gramma seedlings. Agron. J. 70:231-237. 